\title{
Relativistic Theory of Superconductivity: BCS vs. Bogolyubov-de Gennes Approaches
}

\author{
A. Ciechan, A. Donabidowicz and K.I. Wysokiński \\ Institute of Physics, M. Curie-Skłodowska University \\ Radziszewskiego 10, 20-031 Lublin, Poland
}

In this paper we shall present the results of numerical calculations of the superconducting characteristics (temperature dependence of the gap function and specific heat) for few different symmetries of the order parameter in relativistic theory of superconductivity. We shall discuss relevance of the results to describe newly discovered superconductors, particularly those containing heavy elements with strong spin-orbit coupling.

PACS numbers: 74.20.-z, 74.20.Fg, 74.20.Rp

\section{Introduction}

Recently, the renewed interest in the relativistic formulation of the theory of superconductivity has been noted. The generalizations of the Bogolyubov-de Gennes mean field approach [1] and the BCS theory in the Gorkov-Nambu Green functions formulation [2] have been proposed. In both cases one starts with the relativistic Dirac equations [3] for fermions and constructs proper order parameter by making use of the relativistic four-spinors and symmetries. The full set of relativistic order parameters consists of 16 components which can be grouped in 5 different sets behaving as scalar (1 component), four-vector (4 components), pseudoscalar (1 component), axial vector (4 components) and antisymmetric tensor (6 components) [1].

Such an approach is interesting from theoretical point of view but it may also be useful in modelling the behaviour of a number of newly discovered superconductors containing high $Z$ elements. The examples include high temperature superconductors based on $\mathrm{Bi}(Z=83)$ e.g. $\mathrm{Bi}_{2} \mathrm{Sr}_{2} \mathrm{CaCu}_{2} \mathrm{O}_{8}$, $\mathrm{Tl}(Z=81)$ e.g. $\mathrm{Tl}_{2} \mathrm{Ba}_{2} \mathrm{CaCu}_{2} \mathrm{O}_{8}$, or $\mathrm{Hg}(Z=80)$ e.g. $\mathrm{HgBa}_{2} \mathrm{Ca}_{2} \mathrm{Cu}_{3} \mathrm{O}_{8+\delta}$; heavy fermion systems 
$\mathrm{UPt}_{3}, \mathrm{U}_{1-x} \mathrm{Th}_{x} \mathrm{Be}_{13}$ with $\mathrm{U}(Z=92)$, Pt $(Z=78), \mathrm{UGe}_{2}$, URhGe and many others. It is known that description of the normal state of materials with heavy elements $(Z>40)$ requires relativistic effects to be taken into account. Other possible area of application of the general theory comprises nuclear matter, neutron stars, quark-gluon plasma etc.

It is the purpose of this work to calculate basic characteristics of superconducting metal with help of fully relativistic theory. Of particular interest are thermodynamic parameters. In this paper we limit our discussion to the temperature dependence of the gap functions for various symmetries and the corresponding specific heat.

\section{Theory and the results}

The formulation of the relativistic theory of superconductivity by Capelle and Gross [1] starts with the Dirac equation and canonical transformation. They have derived four-component Bogolyubov-de Gennes mean field equations valid for non-homogeneous systems.

The straightforward generalization of the BCS theory of superconductivity has been developed by Ohsaku [2], who started with the Dirac description of the interacting many-body systems and applied the Gorkov-Nambu scheme. The equation for the scalar order parameter takes on the form

$$
1=\frac{g_{0}}{2} \int_{-\Lambda}^{\Lambda} \frac{\mathrm{d}^{3} k}{(2 \pi)^{3}}\left(\frac{1}{2 E_{+}} \tanh \frac{\beta}{2} E_{+}+\frac{1}{2 E_{-}} \tanh \frac{\beta}{2} E_{-}\right) .
$$

Here $\beta=1 / k_{\mathrm{B}} T, g_{0}$ denotes attractive interaction and $\Lambda$ the cut-off parameter. The energy spectrum of relativistic quasiparticles for scalar symmetry of the order parameter $\Delta_{\mathrm{S}}$ reads

$$
E_{ \pm}^{2}=\left(\sqrt{(c \hbar k)^{2}+\left(m c^{2}\right)^{2}} \mp \mu\right)^{2}+\left|\Delta_{\mathrm{S}}\right|^{2},
$$

where $\mu$ stands for the chemical potential. It has to be noted that both the shape of the equation for the gap as well as the quasiparticle spectrum depends on the symmetry of the order parameter [2].

Similarly the structure of the Dirac-Bogolyubov-de Gennes (DBdG) equations explicitly depends on the symmetry. Again, for the scalar order parameter one finds the following equations [1]:

$$
\begin{aligned}
& \gamma^{0}\left(c \boldsymbol{\gamma} \cdot \boldsymbol{p}+m c^{2}+q \gamma^{\nu} A_{\nu}\right) u_{i j k}(\boldsymbol{r})+\int \mathrm{d}^{3} r^{\prime} \Delta_{\mathrm{S}}\left(\boldsymbol{r}, \boldsymbol{r}^{\prime}\right) \eta v_{j k}\left(\boldsymbol{r}^{\prime}\right) \\
& \quad=E_{j k} u_{j k}(\boldsymbol{r}), \\
& -\gamma^{0}\left(c \boldsymbol{\gamma} \cdot \boldsymbol{p}+m c^{2}+q \gamma^{\nu} A_{\nu}\right)^{*} v_{i j k}(\boldsymbol{r})-\int \mathrm{d}^{3} r^{\prime} \Delta_{\mathrm{S}}^{*}\left(\boldsymbol{r}, \boldsymbol{r}^{\prime}\right) \eta u_{j k}\left(\boldsymbol{r}^{\prime}\right) \\
& \quad=E_{j k} v_{j k}(\boldsymbol{r}),
\end{aligned}
$$

where $A_{\nu}=(-\mu / q, \boldsymbol{A})$ and 


$$
\Delta_{\mathrm{S}}\left(\boldsymbol{r}, \boldsymbol{r}^{\prime}\right)=V_{0} \sum_{j k} u_{j k}^{\mathrm{T}}(\boldsymbol{r}) \eta v_{j k}^{*}\left(\boldsymbol{r}^{\prime}\right)\left[1-2 f\left(E_{j k}\right)\right]
$$

and $V_{0}$ denotes (constant) fermion-fermion attraction, $\mu$ chemical potential, $q$ electron charge, $\eta$ is a symmetry matrix, $u_{j k}, v_{j k}$ - four-component quasihole and quasiparticle amplitudes with $j=1,2,3,4$ and $k$ denoting a set of quantum numbers. In translationally invariant system studied below it will be a wave vector denoted by $\boldsymbol{k}$.

Solving either the BCS or DBdG equations for parameters typical of a metal one finds temperature dependence of the gap function, which for scalar symmetry is shown in Fig. 1a and is similar to that found in non-relativistic theory for spinsinglet pairing. On the other hand, the pseudovector symmetry (shown in Fig. 1b) is a generalization of the spin-triplet symmetry.
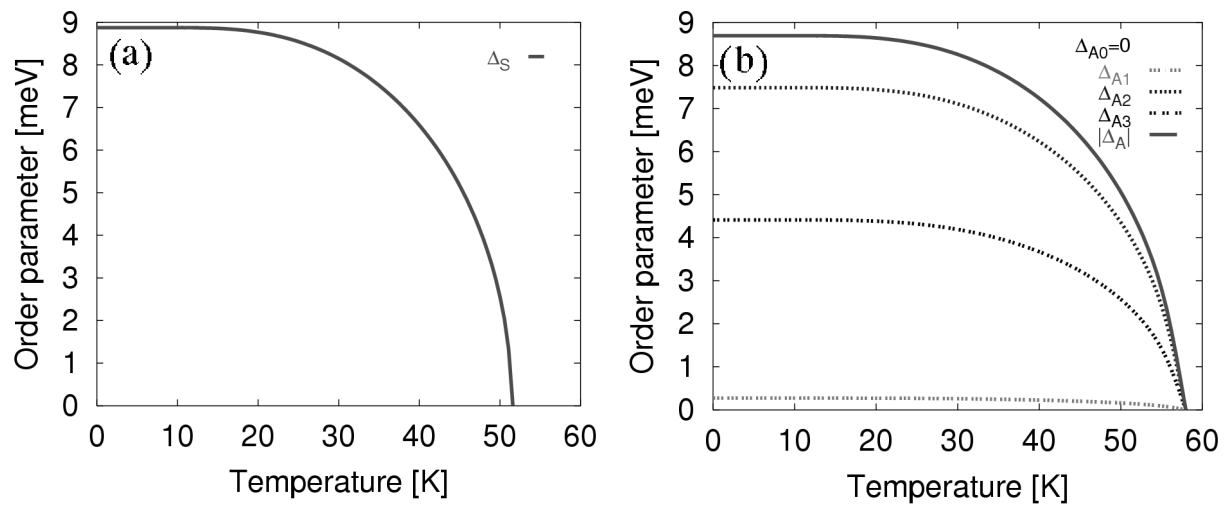

Fig. 1. Temperature dependence of the order parameter for scalar (a) and pseudovector symmetry (b), both calculated for $g_{0}=V_{0}=3.5 \mathrm{eV}$.

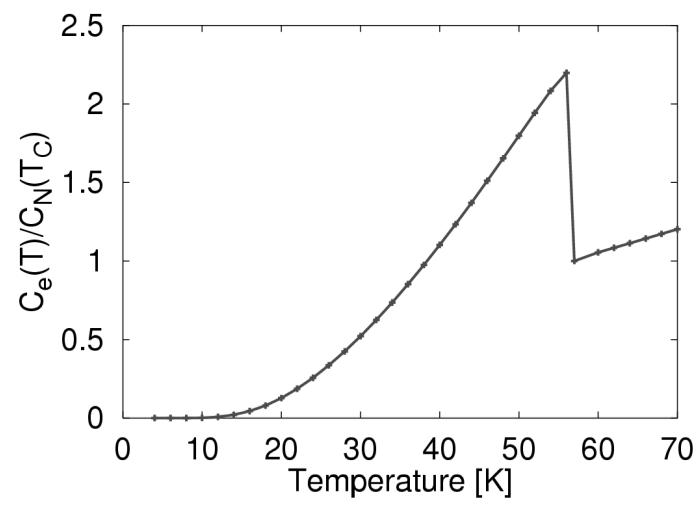

Fig. 2. Temperature dependence of the fermionic specific heat of the relativistic superconductor with scalar symmetry of the order parameter vs. normal state value at $T_{\mathrm{c}}$. 
In the latter case three space components are different from zero, while the time component describing pairing between particle and antiparticle vanishes in a conventional metal.

Specific heat $C_{\mathrm{e}}$ is calculated from the standard quasiparticles entropy formula and takes on the form

$$
C_{\mathrm{e}}=-\frac{1}{k_{\mathrm{B}} T^{2}} \sum_{j \boldsymbol{k}} f\left(E_{j \boldsymbol{k}}\right)\left[1-f\left(E_{j \boldsymbol{k}}\right)\right]\left(E_{j \boldsymbol{k}}^{2}-E_{j \boldsymbol{k}} T \frac{\partial E_{j \boldsymbol{k}}}{\partial T}\right) .
$$

Temperature dependence of $C_{\mathrm{e}}$ for scalar symmetry is shown in Fig. 2 and for a metal it is similar to the predictions of BCS theory.

\section{Summary and conclusions}

The basic properties of the relativistic superconductor were calculated and presented for two out of five possible symmetries. We used parameters typical of a metal and found the behaviour similar to BCS predictions. The relativistic scalar symmetry reduces to $s$-wave spin-singlet superconductor in non-relativistic limit, while the pseudovector reduces to spin-triplet order parameter similar to that found in superfluid ${ }^{3} \mathrm{He}$. The difference between relativistic and non-relativistic characteristics can be in part accounted for by taking spin-orbit coupling into account. It will be of primary importance to find out signs of relativistic symmetries in superconductors containing heavy elements for which relativistic effects are important.

\section{Acknowledgments}

This work was partially supported by the State Committee for Scientific Research grant no. 2P03B 06225.

\section{References}

[1] K. Capelle, E.K.U. Gross, Phys. Rev. B 59, 7140, 7154 (1999); K. Capelle, E.K.U. Gross, B.L. Györffy, Phys. Rev. Lett. 78, 3753 (1997).

[2] T. Ohsaku, Phys. Rev. B 65, 024512 (2001); 66, 054518 (2002).

[3] P. Strange, Relativistic Quantum Mechanics with Application in Condensed Matter and Atomic Physics, Cambridge University Press, Cambridge 1998. 